Validated by the Experiments.

Michael Leguèbe, Aude Silve , Lluis M. Mir , Clair Poignard

\title{
RESEARCH
}

REPORT

$\mathrm{N}^{\circ} 8496$

March 2014

Project-Team MC2 



\title{
Inĩáa
}

\section{Conducting and Permeable States of Cell Membrane Submitted to High Voltage Pulses. Mathematical and Numerical Studies Validated by the Experiments.}

\author{
Michael Leguèbe, Aude Silve ${ }^{*}$, Lluis M. Mir †, Clair Poignard \\ Project-Team MC2
}

Research Report nº 8496 - March 2014 - 25 pages

\begin{abstract}
The aim of this report is to present a new model of in vitro cell electropermeabilization, which describes separately the conducting state and the permeable state of the membrane submitted to high voltage pulses. We first derive the model based on the experimental observations and we present the numerical methods to solve the non-linear partial differential equations. We then present numerical simulations that corroborate qualitatively the experimental data dealing with the uptake of propidium iodide (PI) after millipulses. This tends to justify the validity of our modeling. Forthcoming work will be to calibrate the parameters of the model for quantitative description of the uptake.
\end{abstract}

Key-words: Cell modeling, Non-linear partial differential equations, Finite differences on cartesian grid, Surface diffusion discretization

* Institute for pulsed power and microwave technology, Karlsruhe Institute of Technology, Germany

$\dagger$ CNRS UMR 8203 \& Institut Gustave Roussy, Laboratoire de Vectorologie et Thérapeutiques Anticancéreuses, France.

RESEARCH CENTRE

BORDEAUX - SUD-OUEST

200 avenue de la Vieille Tour

33405 Talence Cedex 


\section{États perméable et conducteur d'une membrane cellulaire soumise à des pulses de fort voltage. Étude mathématique et numérique validée par les expériences.}

Résumé : Le but de ce rapport est de présenter un nouveau modèle d'électroperméabilisation de cellules in vitro, décrivant séparément les états conducteur et perméable de la membrane soumise à des pulses électriques de fort voltage. Nous construisons d'abord le modèle à partir d'observations expérimentales et nous présentons les méthodes numériques permettant de résoudre les équations aux dérivées partielles. Nous présentons ensuite les simulations corroborant qualitativement les données expérimentales sur l'internalisation d'iodure de propidium après plusieurs millipulses. La validité de notre modèle semble ainsi justifiée. Un travail ultérieur consistera en la calibration des paramètres du modèle pour fournir des résultats quantitatifs.

Mots-clés : Modélisation cellulaire, équations aux dérivées partielles non-linéaires, différences finies sur grille cartésienne, discrétisation de diffusion surfacique 


\section{Introduction}

Electroporation is a destructuration of a cell membrane organization leading to an increase of permeability to molecules that usually do not diffuse across the membrane. Even though the increase of membrane permeability is a consequence of the electric shock, the internalization of molecules into the cytoplasm cannot be described by the conducting state of the membrane. More precisely, it has been experimentally observed that the cell membrane may remain permeable several minutes after the electric pulses delivery, while experiments by Benz, Zimmerman et al. 1] have reported that the membrane conductivity almost recovers its steady value within several microseconds after the end of the pulse. Therefore it is important, from the modeling point of view, to distinguish the electric phenomenon, which leads to the increase of membrane conductivity, from the transport of molecules across the permeable membrane. This transport can be obtained by different ways, depending on the molecules: small molecules, which do not interact neither with the membrane and nor with the cytoskeleton can diffuse in the cytoplasm, while active transport (such as ramping process on the membrane or transport due to electrophoretic forces) are needed to make large molecules such as DNA enter the cell.

An electrodiffusion model was already proposed and studied by Smith and Weaver [18, but it is restricted to the $1 \mathrm{D}$ case and the coupling between electroporation and transport across the membrane was not considered. Here we provide a model that describes the in vitro process of the internalization of extracellular molecules into the cell, thanks to the application of high amplitude pulses. Our model is based on a non-linear system of partial differential equations, and the numerical results are obtained for 3-dimensional cells.

Even though it is well-known by experimenters that high conducting state and high permeable state of the membrane do not coincide, the current models of electroporation do not distinguish these two states. For instance, the currently most achieved model of Krassowska and Neu [3. 11, 12, 17. only describes the electrical potential in the cell. Their modeling leads to membrane conducting state, which lasts several seconds (see [3], Fig.7). Such a duration is smaller than the permeable state duration observed by experiments with bleomycin - which still enters the cell several minutes after the end of the pulse - but much longer than the duration of the conducting state of the membrane, which stays highly conducting during several microseconds according to [1]. For all these reasons, the current models were not satisfactory. In this paper, we propose a new model, which differenciates the conducting state from the permeable state of the membrane, and we show that the simulations corroborate the experimental data.

The paper is organized as follows. In the next section, we present generically the system of partial differential equations, which will be used to model the cell electropermeabilization. We then clarify the assumptions on which is based the model, and we derive the non-linear law that account for the change in the conducting and permeable states of the membrane. We then present numerical methods that make it possible to simulate accurately the electric field and the transport of the molecules from the extracellular domain into the cell cytoplasm. We end by numerical simulations that corroborate qualitatively the different experimental observations.

\section{Statement of the generic partial differential equations}

In this section, we briefly present the main partial differential equations that describe the phenomenon. Roughly speaking, it consists of a Poisson equation for the electric potential and a diffusion-transport equation for the non-permeant molecules. In section 3 , we will focus on the non-linearity due to the electropermeabilization. 


\section{$2.1 \quad$ Geometry, notations}

The cytoplasm $\mathcal{O}_{\mathrm{c}}$ and the extracellular medium $\mathcal{O}_{\mathrm{e}}$ are considered as homogeneous material with respective conductivities (see Fig. 1)

$$
\sigma=\left\{\begin{array}{l}
\sigma_{\mathrm{e}} \text { in } \mathcal{O}_{\mathrm{e}}, \\
\sigma_{\mathrm{c}} \text { in } \mathcal{O}_{\mathrm{c}}
\end{array}\right.
$$

We denote by $\Gamma$ the boundary of $\mathcal{O}_{\mathrm{c}}$ which is supposed smooth. Let $\Omega=\mathcal{O}_{\mathrm{e}} \cup \mathcal{O}_{\mathrm{c}} \cup \Gamma$ be the whole domain, and $\partial \Omega$ its boundary.

The membrane is thus described by the single interface $\Gamma$ with no thickness, and $\boldsymbol{\nu}$ designates the unit normal vector to $\Gamma$, outward from $\mathcal{O}_{c}$. The flux of a function $f$ across $\Gamma$ is noted $\left.\partial_{\nu} f\right|_{\Gamma^{+}}$ or $\left.\partial_{\nu} f\right|_{\Gamma^{-}}$depending on the side of the interface, respectively $\mathcal{O}_{\mathrm{e}}$ for $\Gamma^{+}$and $\mathcal{O}_{\mathrm{c}}$ for $\Gamma^{-}$. We use the following notation for the jump of a function $f$ across the interface:

$$
[f]_{\Gamma}=\left.f\right|_{\Gamma^{+}}-\left.f\right|_{\Gamma^{-}} .
$$

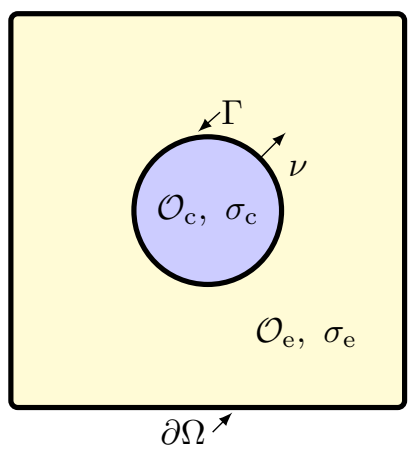

Figure 1: Scheme of the cell embedded in the extracellular domain.

\subsection{Electric potential}

The electric potential is governed by the following equations:

$$
\begin{aligned}
& \Delta u=0, \text { in } \mathcal{O}_{\mathrm{c}} \cup \mathcal{O}_{\mathrm{e}}, \\
& \left.\sigma_{\mathrm{e}} \partial_{\boldsymbol{\nu}} u\right|_{\Gamma^{+}}=\left.\sigma_{\mathrm{c}} \partial_{\boldsymbol{\nu}} u\right|_{\Gamma^{-}}, \\
& \mathrm{C}_{\mathrm{m}} \partial_{t}[u]_{\Gamma}+\mathrm{S}_{0}\left([u]_{\Gamma}-u_{0}\right)+\mathrm{S}_{\mathrm{ep}}\left(t,[u]_{\Gamma}\right)[u]_{\Gamma}=\left.\sigma_{\mathrm{c}} \partial_{\boldsymbol{\nu}} u\right|_{\Gamma^{-}}, \\
& \left.u(t, \cdot)\right|_{\partial \Omega}=u_{\mathrm{imp}}(t, \cdot), \quad u(0, \cdot)=u_{0},
\end{aligned}
$$

where $\mathrm{S}_{0}$ is the resting membrane conductivity, $u_{0}$ is the resting potential and $u_{\text {imp }}$ is the boundary condition determined by the pulse. Equation $\sqrt{1 \mathrm{~b}}$ corresponds to the continuity of the electric current through the membrane. Equation $(1 \mathrm{c})$ is a Kirchhoff law, where the $\mathrm{C}_{\mathrm{m}} \partial_{t}[u]_{\Gamma}$ term represents the capacitive effect of the membrane and $S_{\mathrm{ep}}\left(t,[u]_{\Gamma}\right)[u]_{\Gamma}$ is the electroporation current.

The description of the conducting state of the membrane is obtained by imposing a nonlinear law on $\mathrm{S}_{\mathrm{ep}}$, that will be described in the next section. Note that the term $\mathrm{S}_{\mathrm{ep}}\left(t,[u]_{\Gamma}\right)[u]_{\Gamma}$ corresponds to the electroporation current of Neu, Krassowska et al. [3]:

$$
I_{\mathrm{ep}}=N_{\mathrm{ep}}\left(t,[u]_{\Gamma}\right) i_{\mathrm{ep}}\left([u]_{\Gamma}\right),
$$


after linearization of the current through one pore $i_{\mathrm{ep}}\left([u]_{\Gamma}\right)$. However, we emphasize that the characteristic time of pore creation of Neu and Krassowska's model depends on the membrane voltage instead of being intrinsic to the membrane. Moreover pore density $N_{\text {ep }}$ is not bounded [3] which is hardly defensible from the physical point of view, and therefore we prefer to change it into a sliding-door model given in section 3 .

\subsection{Diffusion and electric transport of non-permeant molecules}

Since the experimental data on electropermeabilization is mainly based on the internalization of non permeant molecules into cells or vesicles, such as propidium iodide (PI) ${ }^{1}$ or DNA, we also describe the motion of these molecules around and inside the cell. This model must take into account the two main modes of propagation of these molecules: the diffusion for small molecules such as PI and the electrophoresis for charged molecules such as DNA. We assume that the electrophoretic forces given by $-\mu_{\mathrm{e}} \nabla u$ holds only in the outer medium, $\mu_{\mathrm{e}}$ being the electrical motility of the molecule $M$ in $\mathcal{O}_{\mathrm{e}}$. This assumption is plausible since the electric field in the cytoplasm is very low due to the shielding effect of the membrane, and since the cytoplasm is composed of cytoskeleton and organels, which prevents the diffusion and the electric transport of large molecules inside the cell.

We suppose that at the initial time, the concentration of $M$ is constant and equal to $M_{0}$ in $\mathcal{O}_{\mathrm{e}}$ while it is set to zero in $\mathcal{O}_{\mathrm{c}}$. Moreover, according to in vitro experiments, the concentration of $M$ on the boundary $\partial \Omega$ of the whole domain $\Omega$ is also constant and equals $M_{0}$. We denote by $\mathrm{d}_{\mathrm{e}}$ and $\mathrm{d}_{\mathrm{c}}$ the diffusion constants of the molecule $M$ in $\mathcal{O}_{\mathrm{e}}$ and $\mathcal{O}_{\mathrm{c}}$ respectively. The concentration $M$ in the outer and in the inner media is governed by the following drift-diffusion equation:

$$
\begin{array}{ll}
\partial_{t} M-\mathrm{d}_{\mathrm{e}} \Delta M=\mu_{\mathrm{e}} \nabla \cdot(M \nabla u), & \text { in } \mathcal{O}_{\mathrm{e}}, \\
\partial_{t} M-\mathrm{d}_{\mathrm{c}} \Delta M=0, & \text { in } \mathcal{O}_{\mathrm{c}},
\end{array}
$$

with the interface conditions on the membrane

$$
\begin{aligned}
& \left.\mathrm{d}_{\mathrm{e}} \partial_{\boldsymbol{\nu}} M\right|_{\Gamma^{+}}+\left.\left.\mu_{\mathrm{e}} M\right|_{\Gamma^{+}} \partial_{\boldsymbol{\nu}} u\right|_{\Gamma^{+}}=\left.\mathrm{d}_{\mathrm{c}} \partial_{\boldsymbol{\nu}} M\right|_{\Gamma^{+}}, \\
& \mathrm{P}_{\mathrm{m}}[M]_{\Gamma}=\left.\mathrm{d}_{\mathrm{c}} \partial_{\boldsymbol{\nu}} M\right|_{\Gamma^{-}}, \\
& \left.M\right|_{t=0}=M_{0} \mathbb{1}_{\mathcal{O}_{\mathrm{e}}},\left.\quad M\right|_{\partial \Omega}=M_{0},
\end{aligned}
$$

where $\mathrm{P}_{\mathrm{m}}$ is the membrane permeability to the considered molecule, and will also be described in the next section. In a similar way as the potential, equation $2 \mathrm{bb}$ states for the flux continuity of $M$ across the membrane. Equation (2c), that expresses the discontinuity of $M$ across $G$, is a Kedem-Kachalsky type of transmission conditions [7].

\section{Electro-poration and electro-permeabilization modeling}

Modeling both membrane poration and permeabilization consists in deriving equations for the surface membrane conductivity $\mathrm{S}_{\mathrm{ep}}$ and the membrane permeability $\mathrm{P}_{\mathrm{m}}$ respectively.

We split the membrane alteration into two different phenomena that occur with two distinct dynamics: the pore creation, with short-term dynamics, and a long-term permeabilization of the lipid bilayer. This splitting is set to account for two experimental results that seem contradictory. On one hand, the observations of Benz et al. 11 and molecular dynamics (MD)

\footnotetext{
${ }^{1} \mathrm{PI}$ is a small molecule which is fluorescent inside the cytoplasm of the cell. It is thus a good fluorescent marker of membrane electropermeabilization.
} 
simulations [19, 21], show that pores shrink within a few microseconds (even a few nanoseconds for MD simulations) after pulses are off. On the other hand, it has been reported that the permeable state lasts several minutes after the pulse delivery [14, 20]. Therefore we differenciate the porated state from the permeabilized state, describing the local degree of poration by $X_{1}$, and respectively the degree of permeabilization by $X_{2}$.

We associate to each state a specific membrane conductivity and membrane permeability:

- $\mathrm{S}_{0}$ and $\mathrm{P}_{0}$ are the respective membrane conductivity and permeability to the molecule $M$ at rest.

- $\mathrm{S}_{1}$ and $\mathrm{P}_{1}$ are the constants that represent the membrane conductivity and the membrane permeability to $M$ of a fully porated region of the membrane,

- $\mathrm{S}_{2}$ and $\mathrm{P}_{2}$ are the membrane conductivity and the membrane permeability to $M$ of the altered lipid bilayer,

The total surface conductivity and permeability of the membrane are then set as

$$
\begin{aligned}
& \mathrm{S}_{\mathrm{m}}(t, s)=\mathrm{S}_{0}+\mathrm{S}_{\mathrm{ep}}(t, s)=\mathrm{S}_{0}+X_{1}(t, s) \mathrm{S}_{1}+X_{2}(t, s) \mathrm{S}_{2}, \quad \forall t>0, s \in \Gamma, \\
& \mathrm{P}_{\mathrm{m}}(t, s)=\mathrm{P}_{0}+X_{1}(t, s) \mathrm{P}_{1}+X_{2}(t, s) \mathrm{P}_{2}, \quad \forall t>0, s \in \Gamma .
\end{aligned}
$$

Let us emphasize the main difference between the membrane conductivity $\mathrm{S}_{\mathrm{m}}$, which is an intrinsic property of the membrane, and $\mathrm{P}_{\mathrm{m}}$, which is the membrane permeability to a specific molecule.

The order of magnitude of $\mathrm{S}_{1}$ is much larger than the resting conductivity $\mathrm{S}_{0}$ as shown in experiments of Benz et al. [1]. Since these observations highlight a remaining conductivity after pulse delivery which is slightly above the resting conductivity, the value $S_{2}$ is set so as

$$
\mathrm{S}_{0}<\mathrm{S}_{2} \ll \mathrm{S}_{1}
$$

Permeabilization constants are taken in the same way,

$$
\mathrm{P}_{0}<\mathrm{P}_{2} \ll \mathrm{P}_{1},
$$

since it is theoretically much easier for a molecule to enter the cytoplasm via a pore rather than through a permeable but non-porated membrane.

Remark 3.1 (A membrane can be simultaneously permeable and not conducting.). Here is the important feature of our modeling: even without any pore, a membrane, which has been fragilised or destructured by the electric pulse has a non-zero permeability, and thus may let molecules enter into the cytoplasm, even though its conductivity is low.

Remark 3.2 (Membrane conductivity is intrinsic, not its permeability ). Note that if the conductivites $\left(\mathrm{S}_{0}, \mathrm{~S}_{1}, \mathrm{~S}_{2}\right)$ are intrinsic to the cell, the permeabilities $\left(\mathrm{P}_{0}, \mathrm{P}_{1}, \mathrm{P}_{2}\right)$ depend on the molecules that cross the membrane, in particular on their molecular weight, spatial conformation and electric charge. For example, if a non-permeant molecule such as bleomycin or DNA is considered, the minimum value of permeability is set to $\mathrm{P}_{0}=0$.

We will now focus on the description of the degree of poration $X_{1}$ in subsection 3.1 and we then describe the degree of permeabilization $X_{2}$ in subsection 3.2 


\subsection{Pore creation and pore resealing}

The function $X_{1}$ describes the degree of porosity of the membrane. It is related to the high conducting state of the membrane as reported by the experiments of Benz et al. [1. It satisfies a differential equation similar to a sliding-door model of electrophysiology. As pores are created only if a threshold voltage is overcome, we set

$$
\partial_{t} X_{1}=F_{1}\left(X_{1},[u]\right),
$$

with the initial condition

$$
X_{1}(t=0, s)=X_{1}^{0} .
$$

The function $F_{1}$ is a function of the transmembrane potential difference $[u]$ and of $X_{1}$ itself given by

$$
F_{1}\left(X_{1},[u]\right)=\frac{\beta_{1}([u])-X_{1}}{\tau_{1}},
$$

where $\tau_{1}$ is the characteristic time of the poration process and $\beta_{1}$ is given by

$$
\beta_{1}(\lambda):=\frac{1+\tanh \left(k_{1}\left(|\lambda|-V_{\mathrm{th}}\right)\right)}{2},
$$

where $k_{1}$ describes the slope of the sigmoidal function and $V_{\text {th }}$ the threshold voltage above which electroporation occurs (see Fig. 2).

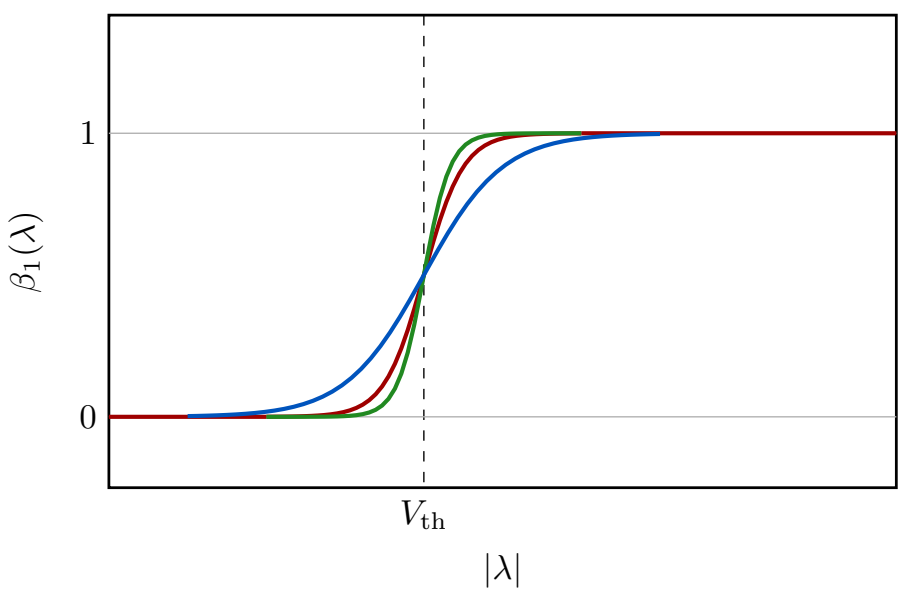

Figure 2: The function $\beta_{1}$ for different slope values $k_{1}$.

\subsection{Membrane permeabilization : a reaction-diffusion model for lipid alteration}

Consider now the degree of permeabilization $X_{2}$. The model of membrane permeabilization is based on the following assumptions, which come from experimental observations:

- We hypothetize that permeabilization results of a long-term effect of defects in the membrane related to an alteration phospholipids due to the presence of water inside the membrane. We thus use $X_{1}$ as an initiating factor of permeabilization. Actually it has been reported by [6, 13] that electric field changes the phospholipid composition, by altering the lipid property. 
- The dynamics of alteration and reconstruction of the membrane are dramatically not the same as it has been observed by the experiments [14, 20. The alteration of the lipids is a physical phenomenon, which occurs as long as pores are present on the membrane and whose characteristic time is in the order of the microsecond. On the contrary, the membrane recovery is a biological phenomenon, called exocytosis, which takes time: it happens for minutes after the electric shock. Thus, we introduce two different time constants: $\tau_{2, \text { perm }}$ for permeabilization and $\tau_{2 \text {,res }}$ for the membrane recovery due to exocytosis, $\tau_{2 \text {,res }}$ being in the order of one hour as reported by Glogauer et al. [5].

- Lipids diffuse along the membrane at a speed $\mathrm{d}_{\mathrm{L}}$ around $1 \mu \mathrm{m}^{2} / \mathrm{s}, 2,23,22$, which is non negligible compared to the lapse of time between two pulses (usually of the order of 1 second), and therefore this surface diffusion has to be accounted for.

We thus use a reaction-diffusion equation to describe the degree of lipid alteration $X_{2}$ :

$$
\partial_{t} X_{2}-\mathrm{d}_{\mathrm{L}} \Delta_{\Gamma} X_{2}=F_{2}\left(X_{1}, X_{2}\right) \text { on } \Gamma,
$$

with the initial condition

$$
X_{2}(t=0, s)=X_{2}^{0}
$$

We set

$$
F_{2}\left(X_{1}, X_{2}\right)=\left\{\begin{array}{l}
\frac{\beta_{2}\left(X_{1}\right)-X_{2}}{\tau_{2, \text { perm }}}, \text { if } \beta_{2}\left(X_{1}\right)-X_{2} \geqslant 0, \\
\frac{\beta_{2}\left(X_{1}\right)-X_{2}}{\tau_{2, \text { res }}}, \text { if } \beta_{2}\left(X_{1}\right)-X_{2} \leqslant 0,
\end{array}\right.
$$

with

$$
\beta_{2}(\lambda):=\frac{1+\tanh \left(k_{2}\left(|\lambda|-X_{1, \mathrm{th}}\right)\right)}{2}
$$

where $X_{1, \text { th }}$ is a poration threshold. Since $F_{2}$ is a Lipschitz function with respect to its first variable, it is clear that existence and uniqueness for $X_{2}$ holds for any $X_{1}$ smooth enough, and $X_{2}$ is also Lipschitz in $X_{1}$. The mathematical analysis of the whole model is not in the scope of this paper and we are confident it can be obtained thanks to quite standard analysis. However, from the modeling point of view it is important to verify that $X_{2}$ is actually a degree of permeabilization, meaning that, similarly to $X_{1}$, it takes values in $[0,1]$. The following proposition ensures this property.

Proposition 3.3 (Boundedness of $X_{2}$ ). Let $X_{2}$ be the solution to (8). Then for almost any $(t, s) \in(0, T) \times \Gamma$

$$
0 \leqslant X_{2}(t, s) \leqslant 1
$$

Proof. Let us define $X_{2}^{-}:=\max \left(0,-X_{2}\right)$. Multiplying $12 \mathrm{k}$ by $X_{2}^{-}$and integrating by part leads to

$$
\frac{1}{2} \frac{d}{d t}\left\|X_{2}^{-}\right\|_{L^{2}(\Gamma)}^{2}+\mathrm{d}_{\mathrm{L}}\left\|\nabla X_{2}^{-}\right\|_{L^{2}(\Gamma)}^{2}=-\int_{\Gamma} F\left(X_{2}, X_{1}\right) X_{2}^{-} d s
$$

but since

$$
F\left(X_{2}, X_{1}\right) X_{2}^{-}=\frac{\beta_{2}\left(X_{1}\right)-X_{2}}{\tau_{2, \text { perm }}} X_{2}^{-} \geqslant 0,
$$


and since $\left.\left\|X_{2}^{-}\right\|^{2}\right|_{t=0}=0$ we infer that $X_{2}$ is positive. Defining $Y_{2}=X_{2}-1$, introducing similarly $Y_{2}^{+}:=\max \left(0, X_{2}-1\right)$, and using the fact that

$$
F_{2}\left(Y_{2}+1, X_{1}\right)=\left\{\begin{array}{l}
\frac{\beta_{2}\left(X_{1}\right)-1-Y_{2}}{\tau_{2, \text { perm }}}, \text { if } \beta_{2}\left(X_{1}\right)-1-Y_{2} \geqslant 0, \\
\frac{\beta_{2}\left(X_{1}\right)-1-Y_{2}}{\tau_{2, \text { res }}}, \text { if } \beta_{2}\left(X_{1}\right)-1-Y_{2} \leqslant 0,
\end{array}\right.
$$

and thus

$$
F\left(Y_{2}+1, X_{1}\right) Y_{2}^{+}=\frac{\beta_{2}\left(X_{1}\right)-1-X_{2}}{\tau_{2, \text { res }}} Y_{2}^{+} \leqslant 0,
$$

shows that $Y_{2}^{+}$equals zero and thus $X_{2} \leqslant 1$.

Remark 3.4 (The choice of the sigmoidal function). For both poration and permeabilization degrees, we used hyperbolic tangents to describe the change in the membrane properties (7)-(10): the functions $\beta_{1}$ and $\beta_{2}$ are defined thanks to two parameters describing a threshold and a speed of the switch between these states. Note that any (smooth enough) sigmoidal function involving similar parameters can be used in the model. In particular, if one wants to relate the degree of poration to the local electrostatic energy, one can use for example:

$$
\beta_{1}(\lambda)=e^{-k\left(\frac{V}{\lambda}\right)^{2}},
$$

with $V$ and $k$ as new threshold and switch speed parameters. However, the hyperbolic tangent has the advantage to identify easily the threshold value and the speed of switch between the nonporated (resp. the non-permeabilized state) state and the porated state (resp. the permeabilized state).

\section{Numerical methods}

Before presenting the numerical methods, let us summarize the complete model of conducting and permeable states of membrane:

$$
\begin{aligned}
& \Delta u=0, \text { in } \mathcal{O}_{\mathrm{c}} \cup \mathcal{O}_{\mathrm{e}}, \\
& \partial_{t} M-\mathrm{d}_{\mathrm{e}} \Delta M=\mu_{\mathrm{e}} \nabla \cdot(M \nabla u), \text { in } \mathcal{O}_{\mathrm{e}}, \\
& \partial_{t} M-\mathrm{d}_{\mathrm{c}} \Delta M=0, \text { in } \mathcal{O}_{\mathrm{c}},
\end{aligned}
$$

with the transmission conditions

$$
\begin{aligned}
& \left.\sigma_{\mathrm{e}} \partial_{\boldsymbol{\nu}} u\right|_{\Gamma^{+}}=\left.\sigma_{\mathrm{c}} \partial_{\boldsymbol{\nu}} u\right|_{\Gamma^{-}}, \\
& \mathrm{C}_{\mathrm{m}} \partial_{t}[u]_{\Gamma}+\mathrm{S}_{0}\left([u]_{\Gamma}-u_{0}\right)+\mathrm{S}_{\mathrm{ep}}\left(t,[u]_{\Gamma}\right)[u]_{\Gamma}=\left.\sigma_{\mathrm{c}} \partial_{\boldsymbol{\nu}} u\right|_{\Gamma^{-}}, \\
& \left.\mathrm{d}_{\mathrm{e}} \partial_{\boldsymbol{\nu}} M\right|_{\Gamma^{+}}+\left.\left.\mu_{\mathrm{e}} M\right|_{\Gamma^{+}} \partial_{\boldsymbol{\nu}} u\right|_{\Gamma^{+}}=\left.\mathrm{d}_{\mathrm{c}} \partial_{\boldsymbol{\nu}} M\right|_{\Gamma^{+}}, \\
& \mathrm{P}_{\mathrm{m}}\left(t,[u]_{\Gamma}\right)[M]_{\Gamma}=\left.\mathrm{d}_{\mathrm{c}} \partial_{\boldsymbol{\nu}} M\right|_{\Gamma^{-}},
\end{aligned}
$$

where

$$
\begin{aligned}
& \mathrm{S}_{\mathrm{ep}}\left(t,[u]_{\Gamma}\right)=X_{1}\left(t,[u]_{\Gamma}\right) \mathrm{S}_{1}+X_{2}\left(t,[u]_{\Gamma}\right) \mathrm{S}_{2}, \\
& \mathrm{P}_{\mathrm{m}}\left(t,[u]_{\Gamma}\right)=X_{1}\left(t,[u]_{\Gamma}\right) \mathrm{P}_{1}+X_{2}\left(t,[u]_{\Gamma}\right) \mathrm{P}_{2},
\end{aligned}
$$

$\mathrm{RR} \mathrm{n}^{\circ} 8496$ 
with

$$
\begin{aligned}
& \partial_{t} X_{1}=F_{1}\left(X_{1},[u]\right), \\
& \partial_{t} X_{2}-\mathrm{d}_{\mathrm{L}} \Delta_{\Gamma} X_{2}=F_{2}\left(X_{1}, X_{2}\right),
\end{aligned}
$$

with the boundary conditions

$$
\left.u\right|_{\partial \Omega}=u_{\mathrm{imp}}(t),\left.\quad M\right|_{\partial \Omega}=M_{0},
$$

and with the initial conditions

$$
u(0, \cdot)=u_{0}, \quad M(0, \cdot)=M^{0} \mathbb{1}_{\mathcal{O}_{\mathrm{e}}}, \quad X_{1}(0, \cdot)=0, \quad X_{2}(0, \cdot)=0 .
$$

\subsection{Discretization of equations in extra- and intracellular domains}

In order to solve numerically the complete model $(12)$, several discretization methods are needed. The equations on the electric potential $u(12 \mathrm{a}),(12 \mathrm{~d})$ and $(12 \mathrm{e})$ are solved using the same numerical scheme as already used in the previous paper dedicated to the electric part of the model [8. We also described precisely the method in [9]. The scheme is based on finite differences on a cartesian grid, with a special treatment of discontinuities at an interface. Its main feature is to insert two additional unknowns per intersection between the interface and the cartesian grid. These unknowns make it possible to compute quantities that are defined on the membrane only, such as $[u], \mathrm{S}_{\mathrm{ep}}$ and $\mathrm{P}_{\mathrm{m}}$. This method is of order 2 in space and order 1 in time. It has been adapted to the 3-dimensional case for the simulations that will be presented in section 5

In order to avoid confusion between the indexation systems that will be described, we will index cartesian grid points by $i$, which is a 3 -uple: $i \in \mathbb{N}^{3}$, while the intersection points between the grid and the interface are denoted by $j \in \mathbb{N}$. Let $\widetilde{\Gamma}$ denote the set of these intersection points.

When necessary, we make the distinction between values inside and outside the cell using the superscripts c and e: $u^{\mathrm{c}}$ and $u^{\mathrm{e}}$ denote the potential respectively inside and outside the cell. $(\Delta u)_{i}^{n}$ and $\left(\partial_{\boldsymbol{\nu}} u^{\mathrm{c}}\right)_{j}^{n},\left(\partial_{\boldsymbol{\nu}} u^{\mathrm{e}}\right)_{j}^{n}$ designate respectively the discretizations of the Laplacian of $u$ at the grid point $P_{i}$ and the normal derivative of $u^{\mathrm{c}}, u^{\mathrm{e}}$ at the point $P_{j}$ of the interface at the time iteration $t^{n}=n \delta t$ ( $\delta t$ being the time pace). The numerical scheme for the potential equations is the following:

$$
\begin{aligned}
\forall i \in & {[0, N]^{3},(\Delta u)_{i}^{n+1}=0, } \\
& \forall P_{j} \in \widetilde{\Gamma}, \sigma_{\mathrm{c}}\left(\partial_{\boldsymbol{\nu}} u^{\mathrm{c}}\right)_{j}^{n+1}-\sigma_{\mathrm{e}}\left(\partial_{\boldsymbol{\nu}} u^{\mathrm{e}}\right)_{j}^{n+1}=0, \\
& \forall P_{j} \in \widetilde{\Gamma}, \frac{\mathrm{C}_{\mathrm{m}}}{\delta t}\left(u_{j}^{\mathrm{e}, n+1}-u_{j}^{\mathrm{c}, n+1}\right)-\sigma_{\mathrm{e}}\left(\partial_{\boldsymbol{\nu}} u^{\mathrm{e}}\right)_{j}^{n+1}=\left(\frac{\mathrm{C}_{\mathrm{m}}}{\delta t}+S_{\mathrm{m}}^{n}\right)\left(u_{j}^{\mathrm{e}, n}-u_{j}^{\mathrm{c}, n}\right),
\end{aligned}
$$

where $N$ is the number of grid discretization points. A Dirichlet boundary condition in a given direction, coupled with an isolating Neumann condition in the other directions, simulate an external uniform electric field around the cell.

Since it is based on the same geometry as the potential, the model of transport and diffusion of molecules is solved with the same discretization method for the Laplacian on $\mathcal{O}_{e}$ and $\mathcal{O}_{c}$, and for the normal derivatives on $\Gamma$. However, since the position of the interface (typically a sphere) in a cartesian grid leads to large irregularities, the method is too restrictive on the time pace to solve equations $12 \mathrm{~b}, 12 \mathrm{c}, 12 \mathrm{f}$ and $12 \mathrm{~g}$ ) in a single iteration. 
We decided to split the transport and diffusion steps as follows: let $\boldsymbol{E}^{n}$ be the electric field computed from $u^{n}$ :

$$
\begin{gathered}
\forall \boldsymbol{i} \in[0, N]^{3}, \quad \frac{1}{\delta t} M_{\boldsymbol{i}}^{*}=\frac{1}{\delta t} M_{\boldsymbol{i}}^{n}+\left(\nabla \cdot\left(\mu M \boldsymbol{E}^{\boldsymbol{n}}\right)\right)_{\boldsymbol{i}}^{n}, \\
\forall P_{j} \in \widetilde{\Gamma}, \quad\left\{\begin{array}{l}
\partial_{\boldsymbol{\nu}} M_{j}^{*}=0 \text { if } \boldsymbol{E}^{n} \cdot \boldsymbol{\nu}>0, \\
M_{j}^{\mathrm{c}, *}=M_{j}^{\mathrm{e}, *} \text { if } \boldsymbol{E}^{\boldsymbol{n}} \cdot \boldsymbol{\nu}<0,
\end{array}\right.
\end{gathered}
$$

where $\left(\nabla \cdot\left(\mu M \boldsymbol{E}^{n}\right)\right)_{i}^{n}$ is the discretization of $\nabla \cdot(\mu M \boldsymbol{E})$ at $P_{\boldsymbol{i}}$ at the time $t^{n}$ using an order 1 upwind scheme, and $\boldsymbol{\nu}$ is the outward unit normal vector to the interface. Note that the motility $\mu$ vanishes if the point $P_{\boldsymbol{i}}$ is inside the cell. The Dirichlet boundary condition equal to $M^{0}$ is used if the electric field is entering the simulation box, that is, when $\nabla u \cdot \boldsymbol{\nu} \geqslant 0$ on $\partial \Omega$.

The diffusion step is discretized as follows

$$
\begin{aligned}
\forall i \in & {[0, N]^{3}, \quad \frac{1}{\delta t} M_{i}^{n+1}-\mathrm{d}(\Delta M)_{i}^{n+1}=\frac{1}{\delta t} M_{i}^{n}, } \\
& \forall P_{j} \in \widetilde{\Gamma}, \quad \mathrm{d}_{\mathrm{e}}\left(\partial_{\nu} M^{\mathrm{e}}\right)_{j}^{n+1}-\mathrm{d}_{\mathrm{c}}\left(\partial_{\nu} M^{\mathrm{c}}\right)_{j}^{n+1}=\mu_{\mathrm{e}} M_{j}^{\mathrm{e}, *}\left(\partial_{\boldsymbol{\nu}} u^{\mathrm{e}}\right)_{j}^{n}, \\
& \forall P_{j} \in \widetilde{\Gamma}, \quad \mathrm{P}_{\mathrm{m}}\left(M_{j}^{\mathrm{e}, n+1}-M_{j}^{\mathrm{c}, n+1}\right)=\mathrm{d}_{\mathrm{e}}\left(\partial_{\boldsymbol{\nu}} M^{\mathrm{e}}\right)_{j}^{n+1},
\end{aligned}
$$

with a homogeneous Neumann condition on the boundary of the simulation box. This numerical scheme is similar to the scheme used in [8] for a static model of electric potential since there is no time derivative in the transmission condition of the discontinuity of $M$. Thus, we use the same fixed point method as in [8]: starting from $M^{k}=M^{n}$, we solve

$$
\begin{aligned}
\forall i \in & {[0, N]^{3}, \quad \frac{1}{\delta t} M_{i}^{k+1}-\mathrm{d}(\Delta M)_{i}^{k+1}=\frac{1}{\delta t} M_{i}^{k}, } \\
& \forall P_{j} \in \widetilde{\Gamma}, \quad \mathrm{d}_{\mathrm{e}}\left(\partial_{\nu} M^{\mathrm{e}}\right)_{j}^{k+1}-\mathrm{d}_{\mathrm{c}}\left(\partial_{\nu} M^{\mathrm{c}}\right)_{j}^{k+1}=\mu_{\mathrm{e}} M_{j}^{\mathrm{e}, *}\left(\partial_{\boldsymbol{\nu}} u^{\mathrm{e}}\right)_{j}^{n}, \\
& \forall P_{j} \in \widetilde{\Gamma}, \quad \mathrm{P}_{\mathrm{m}}\left(M_{j}^{\mathrm{e}, k+1}-M_{j}^{\mathrm{c}, k}\right)=\mathrm{d}_{\mathrm{e}}\left(\partial_{\boldsymbol{\nu}} M^{\mathrm{e}}\right)_{j}^{k+1},
\end{aligned}
$$

until the residual $\left\|M^{k+1}-M^{k}\right\|_{L^{2}(\Gamma)} /\left\|M^{k}\right\|_{L^{2}(\Gamma)}$ is inferior to $10^{-8}$. Then we use $M^{k+1}$ as the solution at the time $t^{n+1}$.

Remark 4.1. Between two pulse deliveries, it is not necessary to solve the equations on the potential, as well as the electrophoretic transport of $M$. It is then possible to use a much larger time step than the step used during the pulses.

\subsection{Discretization of the reaction-diffusion model on the interface}

In order to discretize equation $12 \mathrm{k}$ ) on $X_{2}$, we will use the following numerical scheme:

$$
\frac{1}{\delta t} X_{2}^{n+1}-\mathrm{d}_{\mathrm{L}}\left(\Delta_{\Gamma}\right) X_{2}^{n+1}=\frac{1}{\delta t} X_{2}^{n}+F_{2}\left(X_{2}^{n}, S_{\mathrm{ep}}\right) .
$$

where $\left(\Delta_{\Gamma}\right) X_{2}^{n+1}$ is the approximation Laplace-Beltrami operator (LBO) $\Delta_{\Gamma}$ on the interface at time $t^{n+1}$.

\subsubsection{Existing $\mathrm{LBO}$ discretizations}

In 25, Xu reviewed several finite volumes methods to discretize the Laplace-Betrami operator. Most of them do not converge, but the author proposed two ways to compute the LBO on a 
smooth surface that converge under certain circumstances. We first applied the indirect discretization using an interpolation of the gradients (see sections 3.1 and 4.1 of [25]), on a mesh generated with the intersection points that were defined in the previous section.

Denoting by $\Gamma(\theta, \varphi)$ the parametrization of the sphere of radius $R$ centered at the origin,

$$
\Gamma(\theta, \varphi)=\left(\begin{array}{c}
R \sin \theta \cos \varphi \\
R \cos \theta \cos \varphi \\
R \sin \varphi
\end{array}\right), \quad \forall(\theta, \varphi) \in[0, \pi] \times[0,2 \pi]
$$

the curvature $\overline{\boldsymbol{H}}$ is given by

$$
\overline{\boldsymbol{H}}=\Delta_{\Gamma} \Gamma=-\frac{2}{R} \boldsymbol{\nu}
$$

and thus we measure the convergence by computing

$$
E_{2}:=\left\|\frac{|\boldsymbol{H}-\overline{\boldsymbol{H}}|_{2}}{|\overline{\boldsymbol{H}}|_{2}}\right\|_{L^{2}(\Gamma)} \quad \text { and } \quad E_{\infty}:=\left\|\frac{|\boldsymbol{H}-\overline{\boldsymbol{H}}|_{2}}{|\overline{\boldsymbol{H}}|_{2}}\right\|_{L^{\infty}(\Gamma)},
$$

where $\boldsymbol{H}$ is the numerical approximation of the curvature.

Figure 3 shows that no convergence is achieved, mainly due to the irregularities of the mesh. Indeed, whatever the pace of the cartesian grid, the intersection with a sphere always produces triangles with very heterogeneous dimensions.

In a second time, we use a more regular mesh, generated by subdividing the faces of an icosaedron, so that all mesh triangles have similar dimensions and are nearly equilateral. The method then achieves the order convergence 1.5 for the $\|\cdot\|_{L^{2}(\Gamma)}$ norm but no convergence for the $\|\cdot\|_{L^{\infty}(\Gamma)}$ norm, as shown on figure 4.

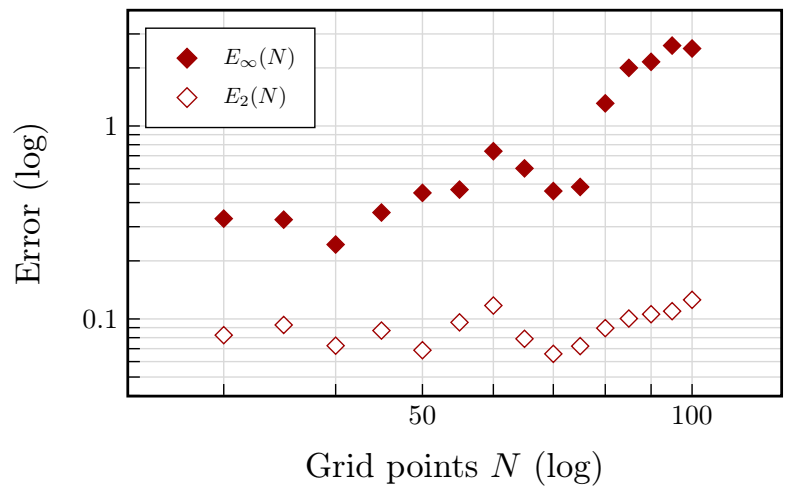

(a)

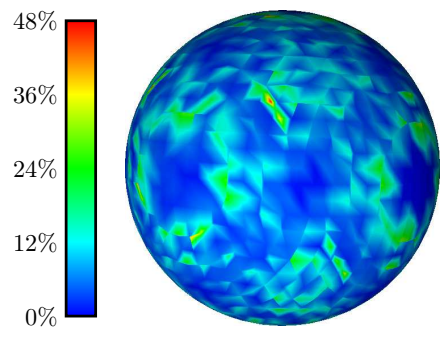

(b)

Figure 3: 3(a). Non-convergence of the LBO discretization given by $\mathrm{Xu}$ in 25 , on a sphere, using the intersection points of $\widetilde{\Gamma}$ between the cartesian grid and the level-set function. The error is defined in equation (18). 3(b) , spatial repartition of $|\boldsymbol{H}-\overline{\boldsymbol{H}}|_{2} /|\overline{\boldsymbol{H}}|_{2}$ on the sphere with a $50^{3}$ points wide cartesian grid. 


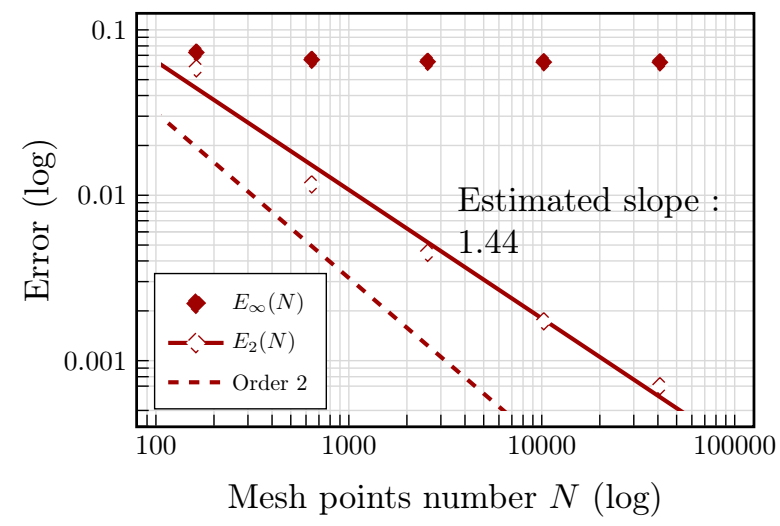

(a)

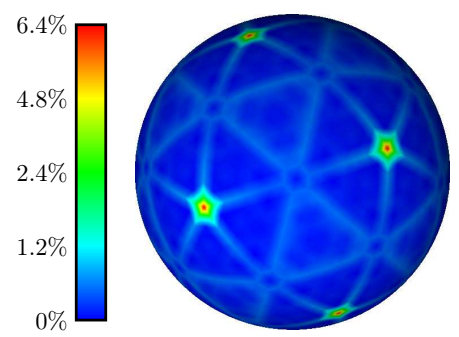

(b)

Figure 4: Convergence 4(a) and spatial repartition of the error 4(b) using a regular mesh of a sphere. This mesh is generated by subdividing the faces of an icosahedron and projecting the vertices on the sphere.

\subsubsection{LBO discretization for parametrized surfaces}

Since a regular mesh is needed to improve convergence, and cells for in vitro experiments usually have a simple shape, we decided to directly express the LBO from a parametrization of the surface $\Gamma$.

Let $\theta \in] 0, \pi[$ and $\varphi \in] 0,2 \pi[$ and $\Gamma=\Gamma(\theta, \varphi)$ be this parametrization. The Riemanian metric at a point $(\theta, \varphi)$ is given by

$$
g_{\theta \theta}:=\left|\partial_{\theta} \Gamma(\theta, \varphi)\right|_{2}^{2}, \quad g_{\varphi \varphi}:=\left|\partial_{\varphi} \Gamma(\theta, \varphi)\right|_{2}^{2}, \quad g_{\theta \varphi}:=\left\langle\partial_{\theta} \Gamma(\theta, \varphi), \partial_{\varphi} \Gamma(\theta, \varphi)\right\rangle
$$

Let

$$
\mathcal{G}:=\left(\begin{array}{cc}
g_{\theta \theta} & g_{\theta \varphi} \\
g_{\theta \varphi} & g_{\varphi \varphi}
\end{array}\right), \quad g:=\operatorname{det}(\mathcal{G}) \quad \text { and } \mathcal{G}^{-1}:=\left(\begin{array}{cc}
g^{\theta \theta} & g^{\theta \varphi} \\
g^{\theta \varphi} & g^{\varphi \varphi}
\end{array}\right)
$$

The LBO is then given by

$$
\Delta_{\Gamma} f(\theta, \varphi)=\frac{1}{\sqrt{|g|}}\left[\partial_{\theta}\left(\sqrt{|g|}\left(g^{\theta \theta} \partial_{\theta} f+g^{\theta \varphi} \partial_{\varphi} f\right)\right)+\partial_{\varphi}\left(\sqrt{|g|}\left(g^{\theta \varphi} \partial_{\theta} f+g^{\varphi \varphi} \partial_{\varphi} f\right)\right)\right]
$$

The interface is discretized by a cartesian grid in $(\theta, \varphi)$, with $N_{\theta}$ points in the $\theta$-direction and $2 N_{\theta}$ points in the $\varphi$-direction, so as the pace $\delta \theta$ is the same in both directions. For the sake of readability, we use the following notations:

$$
\begin{aligned}
& \forall 0 \leqslant k \leqslant N_{\theta}, \theta_{k}:=\left(k+\frac{1}{2}\right) \delta \theta, \quad \forall 0 \leqslant l \leqslant 2 N_{\theta}, \varphi_{l}:=\left(l+\frac{1}{2}\right) \delta \theta, \\
& \widetilde{g}_{k l}:=\sqrt{\left|g\left(\theta_{k}, \varphi_{l}\right)\right|}, \quad g_{k l}^{\alpha \beta}=g^{\alpha \beta}\left(\theta_{k}, \varphi_{l}\right), \quad \forall(\alpha, \beta) \in\{\theta, \varphi\} .
\end{aligned}
$$


The LBO is discretized using a centered second-order finite differences formula:

$$
\begin{aligned}
& \left(\Delta_{\Gamma} f\right)_{k l}=\frac{1}{\widetilde{g}_{k l}}\left[\frac{\widetilde{g}_{k+\frac{1}{2} l} g_{k+\frac{1}{2} l}^{\theta \theta}\left(f_{k+1 l}-f_{k l}\right)-\widetilde{g}_{k-\frac{1}{2} l} g_{k-\frac{1}{2} l}^{\theta \theta}\left(f_{k l}-f_{k-1 l}\right)}{\delta \theta^{2}}\right. \\
& +\frac{\widetilde{g}_{k l+\frac{1}{2}} g_{k l+\frac{1}{2}}^{\varphi \varphi}\left(f_{k l+1}-f_{k l}\right)-\widetilde{g}_{k l-\frac{1}{2}} g_{k l-\frac{1}{2}}^{\varphi \varphi}\left(f_{k l}-f_{k l-1}\right)}{\delta \theta^{2}} \\
& +\frac{\widetilde{g}_{k+1 l} g_{k+1 l}^{\theta \varphi}\left(f_{k+1 l+1}-f_{k+1 l-1}\right)-\widetilde{g}_{k-1 l} g_{k-1 l}^{\theta \varphi}\left(f_{k-1 l+1}-f_{k-1 l-1}\right)}{4 \delta \theta^{2}} \\
& \left.+\frac{\widetilde{g}_{k l+1} g_{k l+1}^{\theta \varphi}\left(f_{k+1 l+1}-f_{k-1 l+1}\right)-\widetilde{g}_{k l-1} g_{k l-1}^{\theta \varphi}\left(f_{k+1 l-1}-f_{k-1 l-1}\right)}{4 \delta \theta^{2}}\right]
\end{aligned}
$$

The following periodicity conditions are used:

$$
\begin{array}{ll}
f_{k, 2 N_{\theta}+1}=f_{k, 0}, & f_{k,-1}=f_{k, 2 N}, \\
f_{-1, l}=f_{0,\left(l+N_{\theta}\right)\left[N_{\theta}\right]}, & f_{N_{\theta}+1, l}=f_{N_{\theta},\left(l+N_{\theta}\right)\left[N_{\theta}\right]} .
\end{array}
$$

Note that the metric has to be computed halfway between grid points, in particular at the poles $\theta=0$ and $\theta=\pi$. Usually, $\mathcal{G}$ is not invertible at these points, and the LBO cannot be defined using the parametrization. In that case, we compute the metric with $\theta=\varepsilon$ or $\theta=\pi-\varepsilon, \varepsilon$ being $\sim 10^{-40}$.

In order to validate our spatial discretization of the LBO, here again we compute the curvature of a sphere. Moreover, we test our discretization on an ellipsoid, whose parametrization is given by:

$$
\Gamma(\theta, \varphi)=\left(\begin{array}{c}
r \sin \theta \cos \varphi \\
r \sin \theta \sin \varphi \\
\alpha r \cos \theta
\end{array}\right), \alpha \neq 0
$$

and the curvature by

$$
\overline{\boldsymbol{H}}=\Delta_{\Gamma} \Gamma(\theta, \varphi)=-\frac{\alpha}{r} \frac{1+\cos ^{2} \theta+\alpha^{2} \sin ^{2} \theta}{\left(\cos ^{2} \theta+\alpha^{2} \sin ^{2} \theta\right)^{2}}\left(\begin{array}{c}
\alpha \sin \theta \cos \varphi \\
\alpha \sin \theta \sin \varphi \\
\cos \theta
\end{array}\right) .
$$

Convergence results for the finite difference method are presented in Fig. 5 and 6 , as expected, the order 2 of convergence is achieved. Remark that convergence is only of order 1 in the case of the ellipsoid for the $\|\cdot\|_{\infty}$ norm because of the correction that is applied at the poles. Compared to the finite volumes methods presented by $\mathrm{Xu}$, we obtain a much better convergence, the drawback being that we need an analytical expression of the surface. 


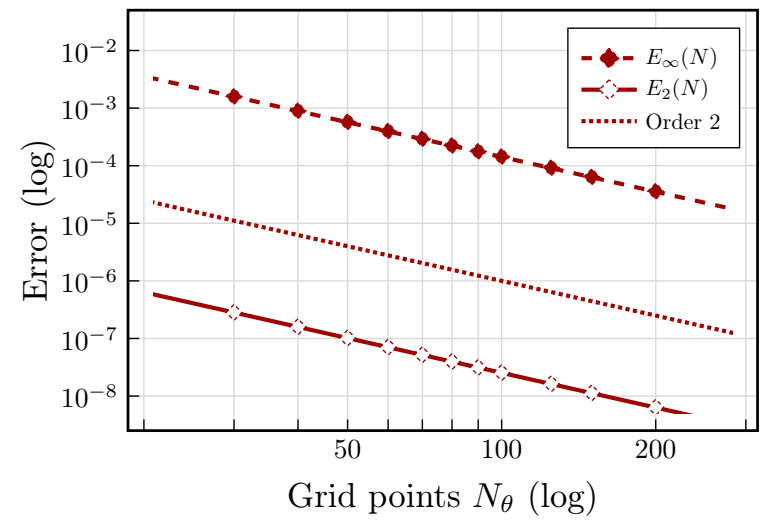

(a)

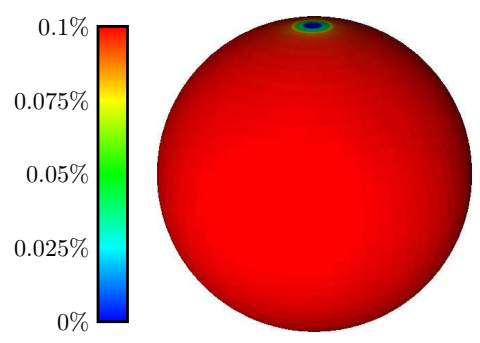

(b)

Figure 5: $5(\mathrm{a})$. Convergence of $(\theta, \varphi)$-LBO-discretization on a sphere. The error is computed by eq. [18). 5(b)] spatial repartition of the error.

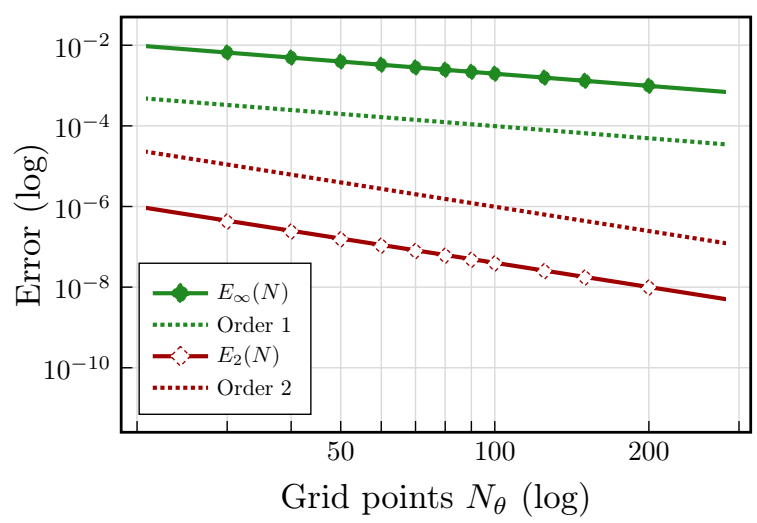

(a)

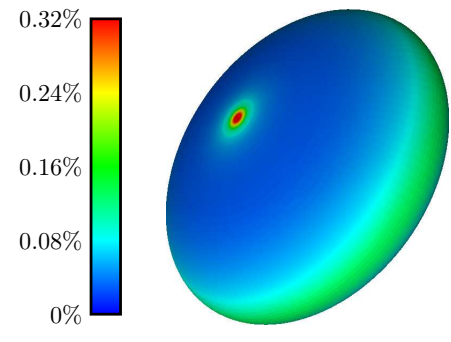

(b)

Figure 6: 6(a) Convergence of the $(\theta, \varphi)$-LBO-discretization on an ellipsoid $(\alpha=0.5)$. The error is computed by eq. [18). 6(b) spatial repartition of the error. 


\subsection{Coupling the discretizations}

In the previous paragraphs, we presented two different ways to describe the interface. Since these two sets of points do not coincide (see fig. 7), we need to perform interpolations between them: once step (13) has been performed, $X_{2}$ must be computed on the $(\theta, \varphi)$-grid to continue on step (17), and the reverse operation has to be done after this diffusion step. In this paragraph, we will designate by intersection points the locations of the intersections between the 3D cartesian grid (representing $\mathcal{O}_{\mathrm{c}} \cup \mathcal{O}_{\mathrm{e}}$ ) and $\Gamma$. The points defined by the $(\theta, \varphi)$-grid involved in the LBO discretization will be called mesh points.

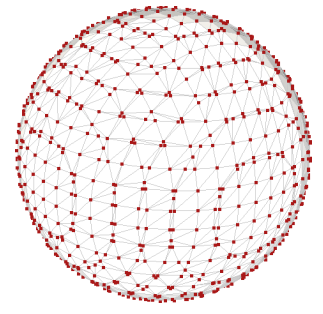

(a)

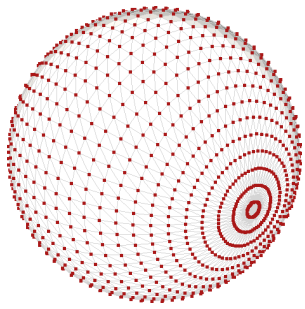

(b)

Figure 7: 7(a) Mesh generated from the intersection points with the cartesian grid, used for the resolution of the potential and transport equations. $7(\mathrm{~b})(\theta, \varphi)$-mesh on which the LBO is discretized.

\subsubsection{From the mesh points to the intersection points}

To obtain the values on the intersection points knowing the function on the mesh points, the coordinates of the intersection points are directly projected on the $(\theta, \varphi)$-grid. This can be done straightforwardly if the expression of the reciprocal parametrization is known. A regular bilinear interpolation is then possible on this grid: if $\left(\theta_{j}, \varphi_{j}\right)$ are the coordinates of the point $P_{j} \in \widetilde{\Gamma}$ in $\left[\theta_{k}, \theta_{k+1}\right] \times\left[\varphi_{l}, \varphi_{l+1}\right]:$

$$
\begin{aligned}
f\left(\theta_{j}, \varphi_{j}\right) \sim & \left(f_{k l}-f_{k+1 l}-f_{k l+1}+f_{k+1 l+1}\right) \frac{\left(\theta_{j}-\theta_{k}\right)\left(\varphi_{j}-\varphi_{l}\right)}{d \theta^{2}} \\
& +\left(f_{k+1 l}-f_{k l}\right) \frac{\theta_{j}-\theta_{k}}{d \theta}+\left(f_{k l+1}-f_{k l}\right) \frac{\varphi_{j}-\varphi_{l}}{d \theta}+f_{k l} .
\end{aligned}
$$

\subsubsection{From the intersection points to the mesh points}

For the reverse interpolation, we consider the 3D-cartesian cell in which is located a mesh point. In this cell, the interface is described by a convex polygon whose vertices are intersection points where values are known (see Fig 8). We use barycentric coordinates to perform the interpolation on the mesh point, as given by Meyer et al. in [10]. Let $P_{j}, j=1, \cdots, j_{\max }$ be the list of these vertices, ordered along $j$ around the mesh point $P_{k l}$. We define the weights

$$
\alpha_{j}=\frac{\operatorname{cotan}\left(\overrightarrow{P_{j} P_{k l}}, \overrightarrow{P_{j} P_{j-1}}\right)+\operatorname{cotan}\left(\overrightarrow{P_{j} P_{k l}}, \overrightarrow{P_{j} P_{j+1}}\right)}{\left|\overrightarrow{P_{k l} P_{j}}\right|_{2}^{2}} .
$$

The value of a function $f$ at $P_{k l}$ is then given by $\sum_{i=1}^{j_{\max }} \alpha_{j} f_{j}$.

Figure 9 shows that the two interpolation methods are of order 2 . 


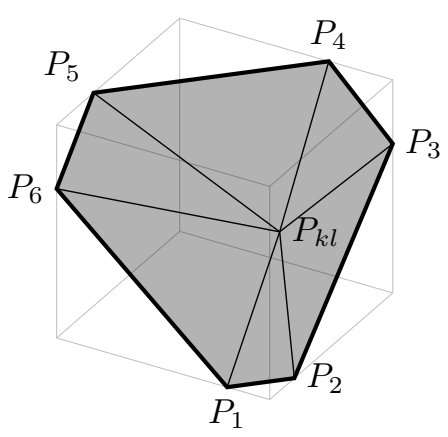

Figure 8: Interpolating values known on the intersection points $P_{j}, j=1: 6$ to evaluate a function at the mesh point $P_{k l}$.

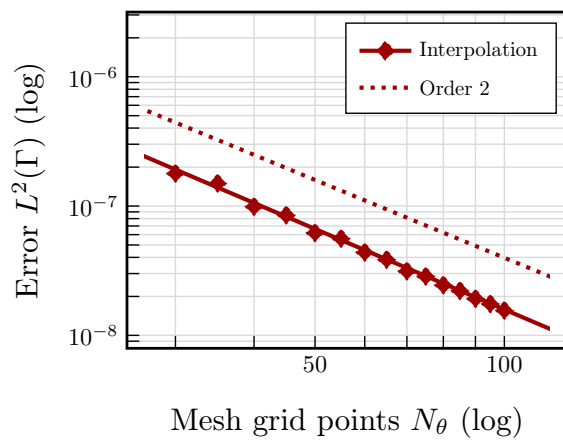

(a)

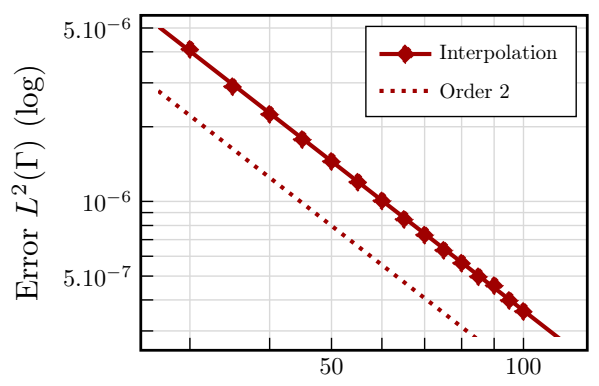

3 D grid points $N(\log )$

(b)

Figure 9: Convergence of interpolation methods for the mapping

$$
(x, y, z) \mapsto x^{2}+1 .
$$

The computed error is the $L^{2}(\Gamma)$ norm of the relative difference between the interpolation and the solution. 9(a) from the mesh points to the intersection points. 9(b) from the intersection points to the mesh points. 


\section{Results}

In order to run the simulations, we need to adjust the parameters of the equations. Parameters for the electric potential can be fixed in accordance with the papers of Neu et al. Moreover, the diffusion of lipids along the membrane is quite well-known, and of order $1 \mu \mathrm{m}^{2} / \mathrm{s}$ and the time constants $\tau_{2 \text {,perm }}$ and $\tau_{2 \text {,res }}$ can be obtained by the experiments. It thus remains to choose the 4 parameters of the sigmoidal functions $\beta_{1}$ and $\beta_{2}$ as well as the values of the permeability $\mathrm{P}_{1}$ and $\mathrm{P}_{2}$. Since it is not the scope of the present to calibrate precisely the model with some specific experiments, we choose parameters that provide results that are qualitatively in accordance with the experiments. All the parameters are given in the table 1 . We emphasize that a precise calibration of the model would be necessary to obtain quantitative results, however this represents a huge amount of work: it needs the development of specific numerical tools and appropriate experimental data to fit with and it is far beyond the scope of the present paper.

\subsection{Diffusion and transport of molecules in a $2 \mathrm{D}$ cell, without lipid diffusion}

In order to validate our model, we first confront both the numerical transport and diffusion of the molecule to the experimental results of PI uptake for various pulses. Based on the articles of Escoffre et al. [4] and Krassowska et al. 3], we choose the parameters for the simulations given by Table $\mathbb{1}^{2}$. We compare the results of the simulation with the experimental data of [4] involving millipulses. Comparison is also led with the observations of Vernier et al. 24], using micro- and nanopulses. In these 2D simulations, the omit the surface diffusion of lipids on the cell membrane.

For long duration pulses, the electrophoretic effect brings more PI on the part of the cell which faces the anode. This accumulation leads to an assymetry in the PI repartition inside the cytosol. Figures 10 and 11 show that the modeling is in good agreement with the experiments, at least qualitatively.

\footnotetext{
${ }^{2}$ Since the time scales are from a few microseconds for the poration to a few hour for the total recovery of the membrane, the computation times are very huge. Since our goal is to provide qualitative behaviours of the model, and not quantitative results, for the sake of simplicity we decrease to $60 \mathrm{~s}$ the time recovery of the membrane by exocytosis. Forthcoming works of parallel computing will be addressed to fit quantitatively the model with the biological data.
} 


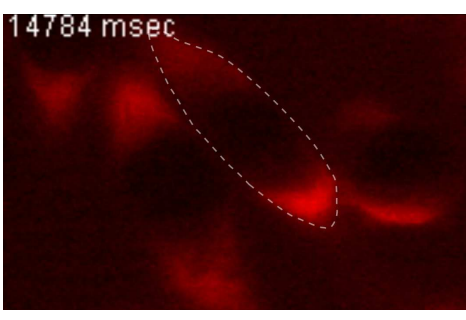

(a) Exp. data of Escoffre et al. 4,
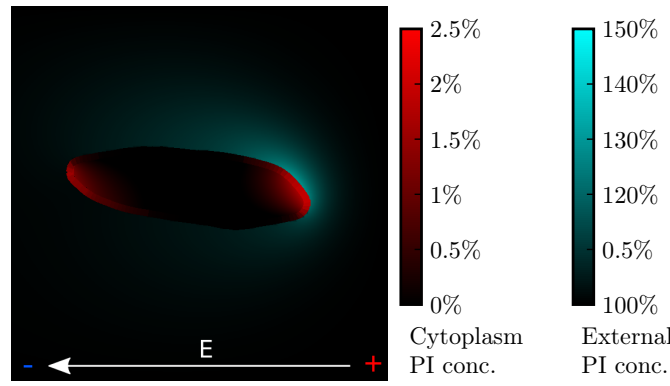

(b) Numerical Simulations with parameters of Table 1

Figure 10: PI uptake during 10 millipulses of $20 \mathrm{~ms}, 50 \mathrm{kV} / \mathrm{m}, 1 \mathrm{~Hz}$. Comparison between the experiments Fig. 10(a), as given by Escoffre et al. [4] and the simulation Fig. 10(b). PI concentrations are given relatively to the external initial concentration. Two different color scales are used to represent PI concentration, since the proportion of PI inside the cell is much lower than outside.
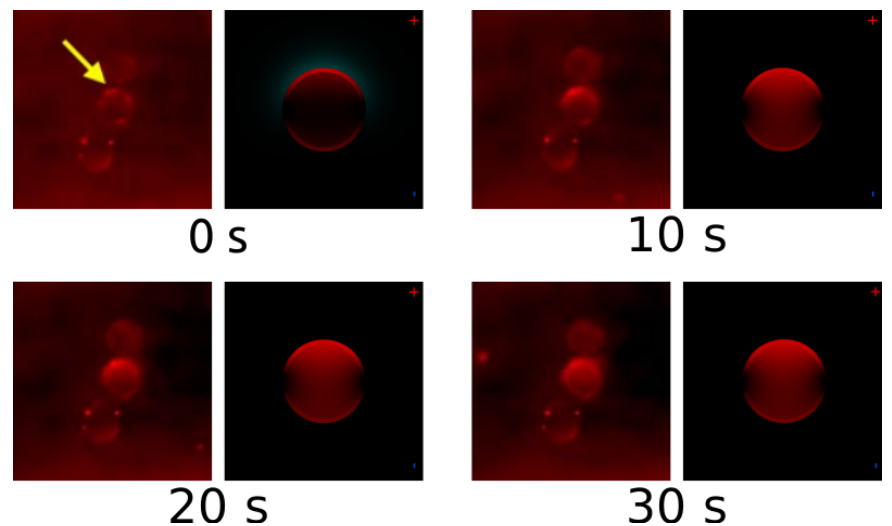

(a) PI uptake with micropulses

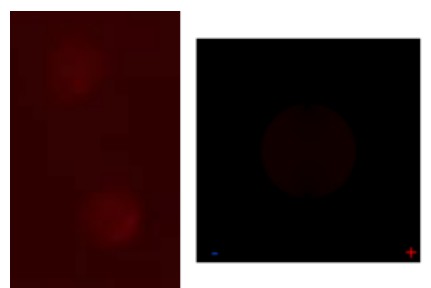

$15 \mathrm{~s}$

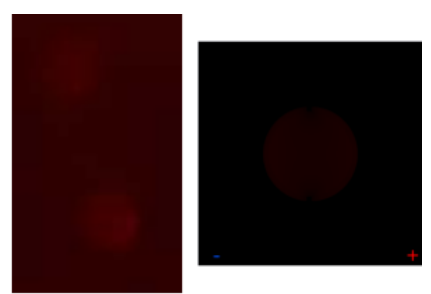

$30 \mathrm{~s}$

(b) PI uptake with nanopulses

Figure 11: Comparison between simulations and experiences from Vernier et al. 24]. The time indications are taken after all pulses are applied. 11(a): 5 micropulses of $100 \mu \mathrm{s}, 500 \mathrm{kV} / \mathrm{m}, 4 \mathrm{~Hz}$. 11(b) : 10 nanopulses of $30 \mathrm{~ns}, 2.5 \mathrm{MV} / \mathrm{m}, 4 \mathrm{~Hz}$. The color scale is the same as in Figure 10. 
Table 1: Simulation parameters. Biological parameters are taken from [3, 4].

\begin{tabular}{|c|c|c|c|}
\hline Variable & Symbol & Value & Unit (SI) \\
\hline \multicolumn{4}{|l|}{ Biological parameters } \\
\hline Spherical cell radius & $r$ & $8 \times 10^{-6}$ & $\mathrm{~m}$ \\
\hline Extracellular conductivity & $\sigma_{\mathrm{e}}$ & 5 & S.m ${ }^{-1}$ \\
\hline Intracellular conductivity & $\sigma_{\mathrm{c}}$ & 0.455 & S.m ${ }^{-1}$ \\
\hline Capacitance & $\mathrm{C}_{\mathrm{m}}$ & $9.5 \times 10^{-3}$ & F. $\mathrm{m}^{-2}$ \\
\hline Membrane surface conductivity & $\mathrm{S}_{0}$ & 1.9 & S.m ${ }^{-2}$ \\
\hline Resting potential & $u_{0}$ & $-40 \times 10^{-3}$ & $\mathrm{~V}$ \\
\hline Molecule diffusion in cytosol & $\mathrm{d}_{\mathrm{c}}$ & $10^{-9}$ & $\mathrm{~m}^{2} \cdot \mathrm{s}^{-1}$ \\
\hline Molecule diffusion in outer medium & $d_{e}$ & $10^{-8}$ & $\mathrm{~m}^{2} \cdot \mathrm{s}^{-1}$ \\
\hline Molecule motility in outer medium & $\mu_{\mathrm{e}}$ & $10^{-6}$ & $\mathrm{~m}^{2} \cdot \mathrm{V}^{-1} \cdot \mathrm{s}^{-1}$ \\
\hline \multicolumn{4}{|l|}{ Model parameters for $X_{1}$} \\
\hline Pore conductivity & $\mathrm{S}_{1}$ & $1.1 \times 10^{6}$ & S. $\mathrm{m}^{-2}$ \\
\hline Pore permeability & $\mathrm{P}_{1}$ & $10^{-6}$ & $\mathrm{~m} \cdot \mathrm{s}^{-1}$ \\
\hline Poration threshold & $V_{\mathrm{th}}$ & 0.2 & $\mathrm{~V}$ \\
\hline Poration switch speed & $k_{1}$ & 40 & $\mathrm{~V}^{-1}$ \\
\hline Poration characteristic time & $\tau_{1}$ & $2 \times 10^{-5}$ & s \\
\hline \multicolumn{4}{|l|}{ Model parameters for $X_{2}$} \\
\hline Altered membrane conductivity & $\mathrm{S}_{2}$ & $10^{3}$ & S.m ${ }^{-2}$ \\
\hline Altered membrane permeability & $\mathrm{P}_{2}$ & $10^{-7}$ & $\mathrm{~m} \cdot \mathrm{s}^{-1}$ \\
\hline Conductivity threshold & $X_{1, \text { th }}$ & $8 \times 10^{4}$ & S.m $\mathrm{m}^{-2}$ \\
\hline Permeabilization switch speed & $k_{2}$ & 10 & $\mathrm{~S}^{-1} \cdot \mathrm{m}^{2}$ \\
\hline Permeabilization dynamic & $\tau_{2, \text { perm }}$ & $10^{-6}$ & $\mathrm{~s}$ \\
\hline Membrane recovery time & $\tau_{2, \text { res }}$ & 60 & $\mathrm{~s}$ \\
\hline
\end{tabular}




\subsection{Simulating the whole model in a $3 \mathrm{D}$ cell}

It has been very recently reported in [16] that, strikingly, for the same number of pulses, a high frequency rate of repetition is less efficient than pulses repeted at low frequency. From the modeling point of view, this question of such "desensitization" has never been addressed and we show in this section that our model can provide an explanation to these observations.

We performed 3D-simulations of a spherical cell submitted to 10 permeabilizing micropulses $(10 \mu \mathrm{s}, 40 \mathrm{kV} / \mathrm{m})$, with various repetition rates from 1 to $1000 \mathrm{~Hz}$. In these simulations, we set the diffusion of the lipids on the membrane to

$$
\mathrm{d}_{\mathrm{L}}=10^{-12} \mathrm{~m}^{2} \mathrm{~s}^{-1} \text {. }
$$

The average permeabilization $\mathrm{P}_{\mathrm{m}}$ of the membrane, as well as the concentration of molecules that entered the cell are measured along time.

Figure 12 shows the distribution of $\mathrm{P}_{\mathrm{m}}$ on the surface of the cell at different instants of the $1 \mathrm{~Hz}$ and $1000 \mathrm{~Hz}$ simulations. A comparative animation of these two simulations is also avalaible as supplementary material. We see that in the case of a fast repetition rate, the altered lipids do not have time to be evenly spread on the membrane. Since the next pulse will alter the same region as the first, the total quantity of altered lipids will be lower than the $1 \mathrm{~Hz}$ case.

Figure 13 presents the average of $\mathrm{P}_{\mathrm{m}}$ after each pulse. As expected, the permeabilization is more efficient if enough time is left between pulses to let the lipids diffuse.

In Figure 14, we plot the average concentration of molecules in the cytoplasm along time, growing as long as the value $\mathrm{P}_{\mathrm{m}}$ is non-zero. We can see that the $1000 \mathrm{~Hz}$ case leads to a lower efficiency of the permeabilization leading to a lower amount of internalized molecules. We emphasize on the fact that the final quantity of molecules is highly dependent on the constants $\tau_{2, \text { res }}$ and $\mathrm{P}_{2}$. If $\mathrm{P}_{2}$ is large enough (for example for very small molecules), the concentration can reach its maximum value in a very short time whatever the pulse frequency. On the contrary, small values of $\mathrm{P}_{\mathrm{m}}$ increase the difference between the final internalized quantity of molecules. In particular, these simulations corroborate results of High Voltage/Low Voltage experiments [15] that, within the first seconds after the pulses, show a better permeabilization to DNA when the lapse of time between pulses is longer. 


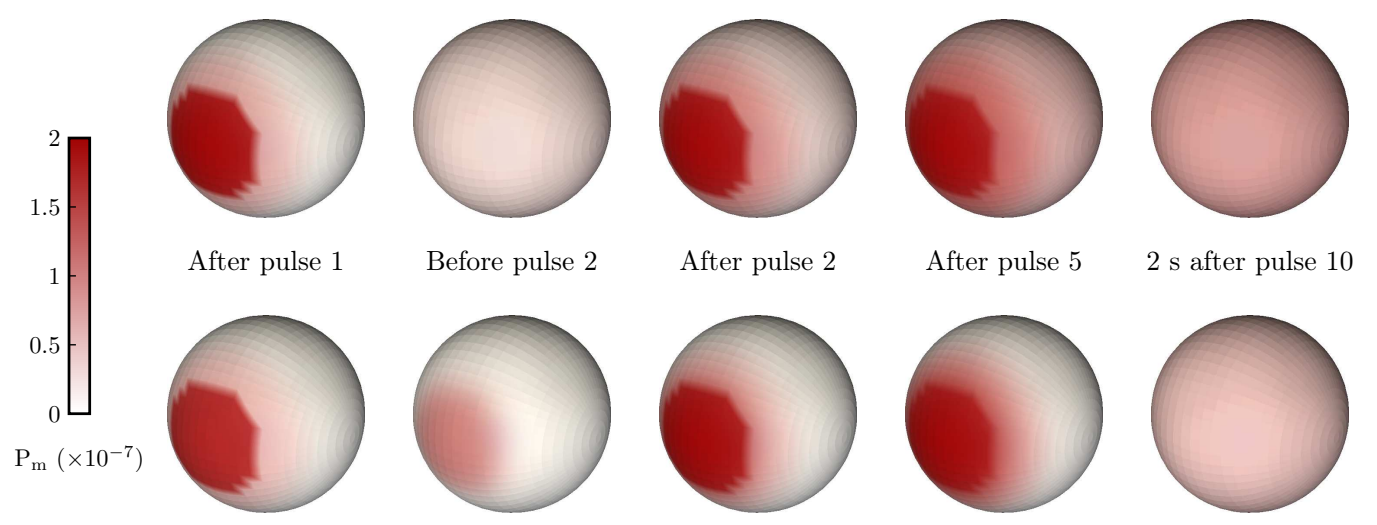

Figure 12: Influence of the pulse frequency on the membrane permeabilization $\mathrm{P}_{\mathrm{m}}$. The magnitude of each pulse is $40 \mathrm{kV} / \mathrm{m}$ during $10 \mu \mathrm{s}$. 10 pulses are applied on both cells, but the time between pulses is different : 1 second for the top line, 1 millisecond for the bottom line. After 10 pulses, the average of $\mathrm{P}_{\mathrm{m}}$ is around $8 \times 10^{-8}$ for the $1 \mathrm{~Hz}$ case, and half for the $1000 \mathrm{~Hz}$ case. An animation of these simulation is available as supplementary content.

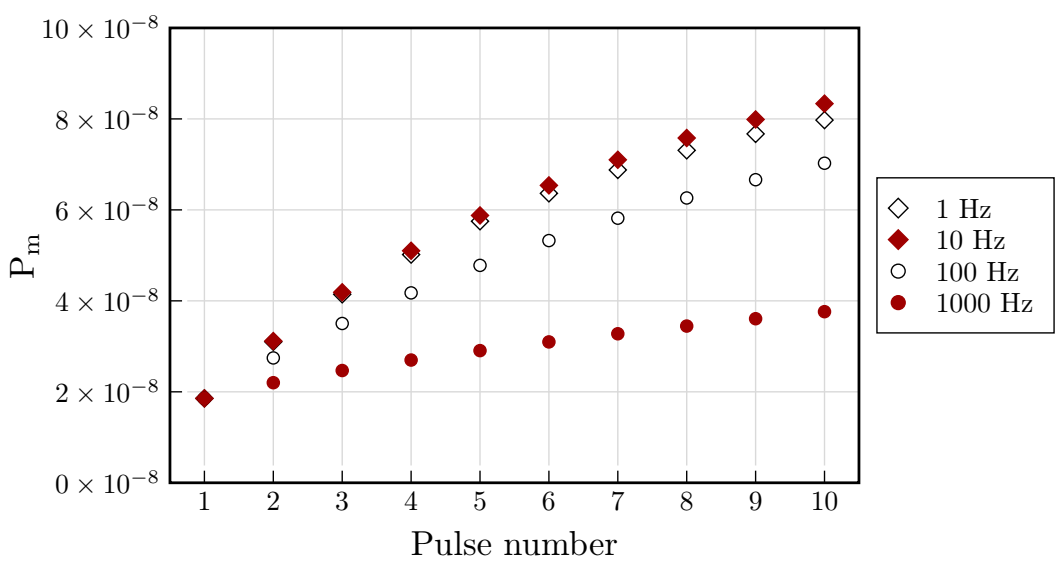

Figure 13: Average permeabilization $\mathrm{P}_{\mathrm{m}}$ on the cell after each of the 10 pulses of Figure 12 for different pulse repetition rates. 


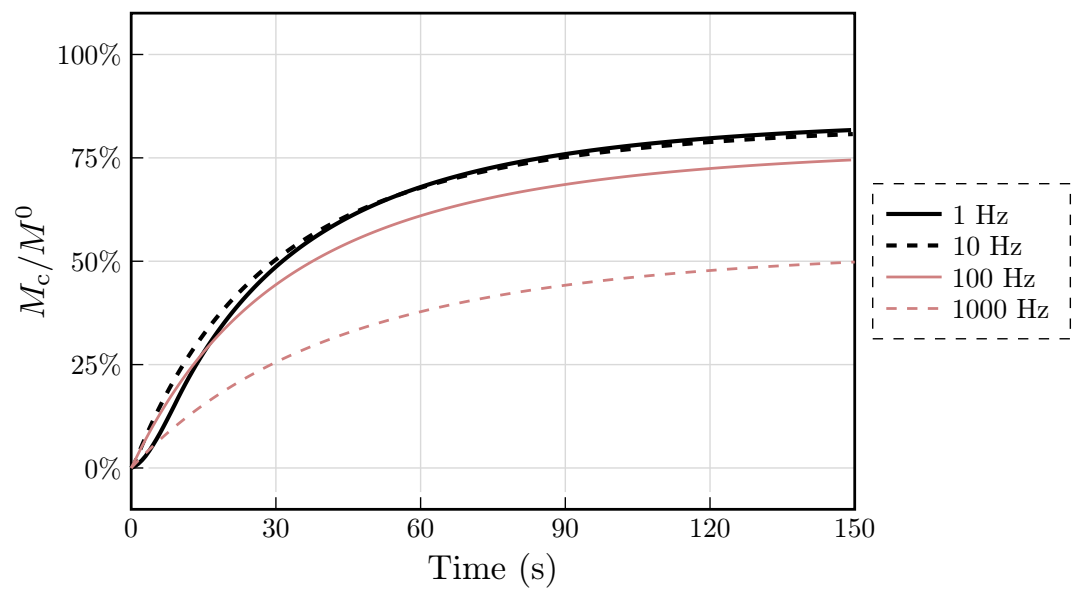

Figure 14: Average concentration $M_{\mathrm{c}}$ inside the cell for different pulses repetition rates, expressed as a percentage of external concentration $M^{0}$. As in Figure 12 and Figure 13,10 micropulses $(40 \mathrm{kV} / \mathrm{m}$ during $10 \mu \mathrm{s}$ each) are applied at different frequencies.

\section{Conclusion}

We have presented a model, which describes simultaneously the conducting and the permeable states of the membrane, without identifying these states. This is an important novelty in the modeling of cell electropermeabilization, which only dealt with the electrical behaviour of the membrane before. Our model makes it possible to compare straightforwardly the majority of the available experimental data, which essentially deal with the diffusion of non permeant molecules across the membrane, taking into account the fast time to recover a low conductivity and the long-time permeabilized state. Another important feature of our model is that the diffusion of the lipids along the membrane makes it possible to explain the striking experimental observations: the more you wait between the pulse, the more efficient is the permeabilization, which cannot be accounted for by considering the membrane conductivity only.

Therefore our numerical results show that the model behaves qualitatively in accordance with the experiments. In order to provide quantitative results, forthcoming work will be to calibrate the parameters. We emphasize that our model was built with the least parameters as possible in order to solve the inverse problem of the fitting.

\section{Acknowledgements}

This study has been carried out in the frame of "the Investments for the future" Programme IdEx Bordeaux â CPU (ANR-10-IDEX-03-02). The authors have been partly granted by the French national agency throughout the research projects INTCELL (2010-BLAN-916-04) and MEMOVE (2011-BS01-006-01). This work was partly performed in the scope of the European Associated Laboratory EBAM. 


\section{References}

[1] R. Benz, F. Beckers, and U. Zimmermann. Reversible electrical breakdown of lipid bilayer membranes: a charge pulse relaxation study. J. Memb. Biol., 48(2):181-204, 1979.

[2] Y. Chen, B.C. Lagerholm, B. Yang, and K. Jacobson. Methods to measure the lateral diffusion of membrane lipids and proteins. Methods, 39(2):147-153, 2006.

[3] K. DeBruin and W. Krassowska. Modelling electroporation in a single cell. I. Effects of field strength and rest potential. Biophys. J., 77(3):1213-1224, 1999.

[4] J.M. Escoffre, T. Portet, C. Favard, J. Teissié, D. Dean, and M.P. Rols. Electromediated formation of DNA complexes with cell membranes and its consequences for gene delivery. BBA - Biomembranes, 1808(6):1538-1543, 2011.

[5] M. Glogauer, W. Lee, and C.A.G. McCulloch. Induced endocytosis in human fibroblasts by electrical fields. Exp. Cell Res., 208(1):232 - 240, 1993.

[6] S. Harakawa, N. Inoue, T. Hori, K. Tochio, T. Kariya, K. Takahashi, F. Doge, H. Suzuki, and H. Nagasawa. Effects of a $50 \mathrm{hz}$ electric field on plasma lipid peroxide level and antioxidant activity in rats. Bioelectromagnetics, 26(7):589-594, 2005.

[7] M. Kargol. A more general form of Kedem and Katchalsky's practical equations. J. Biol. Phys., 22(1):15-26, 1996.

[8] O. Kavian, M. Leguèbe, C. Poignard, and L. Weynans. "Classical" Electropermeabilization Modeling at the Cell Scale. J. Math. Biol., 68(1-2):235-265, 2014.

[9] M. Leguèbe, C. Poignard, and L. Weynans. A second-order Cartesian method for the simulation of electropermeabilization cell models. Research report RR-8302, INRIA, 2013.

[10] M. Meyer, A. Barr, H. Lee, and M. Desbrun. Generalized barycentric coordinates on irregular polygons. J. Graph. Tools, 7(1):13-22, 2002.

[11] J. Neu and W. Krassowska. Asymptotic model of electroporation. Phys. Rev. E, 53(3):3471$3482,1999$.

[12] J. Neu and W. Krassowska. Singular perturbation analysis of the pore creation transient. Phys. Rev. E, 74(031917):1-9, 2006.

[13] B. Nikolova, M. Georgieva, D. Savu, and I. Tsoneva. Cell membrane alteration by weak alternating electric field at low frequency. Rom. Rep. Phys., 64(4):1046-1052, 2012.

[14] M.P. Rols, C. Delteil, M. Golzio, and J. Teissié. Control by ATP and ADP of voltageinduced mammalian-cell-membrane permeabilization, gene ransfer and resulting expression. Eur. J. of Biochem., 254(2):382-388, 1998.

[15] S. Šatkauskas, F.M. André, M.F. Bureau, D. Scherman, D. Miklavčič, and L.M. Mir. Electrophoretic component of electric pulses determines the efficacy of in vivo DNA electrotransfer. Hum. Gene Ther., 16(10):1194-1201, 2005.

[16] A. Silve, A. Giumerà Brunet, A. Ivorra, and L.M. Mir. Comparison of the effects of the repetition rate between microsecond and nanosecond pulses: Electropermeabilisation-induced electro-desensitization? Submitted, 2014. 
[17] K.C. Smith, J. Neu, and W. Krassowska. Model of creation and evolution of stable electropores for DNA delivery. Biophys. J., 86(5):2813-2826, 2004.

[18] K.C. Smith and J.C. Weaver. Electrodiffusion of molecules in aqueous media: a robust, discretized description for electroporation and other transport phenomena. IEEE T. Biomed. Eng., 59(6):1514-1522, 2012.

[19] M. Tarek. Membrane Electroporation: A Molecular Dynamics Simulation. Biophys. J., 88(6):4045-4053, 2005.

[20] J. Teissié and C. Ramos. Correlation between Electric Field Pulse Induced Long-Lived Permeabilization and Fusogenicity in Cell Membranes. Biophys. J., 74(4):1889-1898, 1998.

[21] D.P. Tieleman. The molecular bases of electroporation. BMC Biochem., 5(10):1-12, 2004.

[22] J.-F. Tocanne, L. Dupou-Cézanne, and A. Lopez. Lateral diffusion of lipids in model and natural membranes. Prog. Lip. Res., 33(3):203-237, 1994.

[23] W.L.C. Vaz, F. Goodsaid-Zalduondo, and K. Jacobson. Lateral diffusion of lipids and proteins in bilayer membranes. FEBS Lett., 174(2):199-207, 1984.

[24] T. Vernier, Y. Sun, L. Marcu, S. Salemi, C.M. Craft, and M.A. Gundersen. Calcium bursts induced by nanosecond electric pulses. Biochemical and Biophysical Research Communications, 310(2):286-295, 2003.

[25] G. Xu. Discrete Laplace-Beltrami operators and their convergence. Comput. Aided Geom. Des., 21(8):767-784, 2004. 


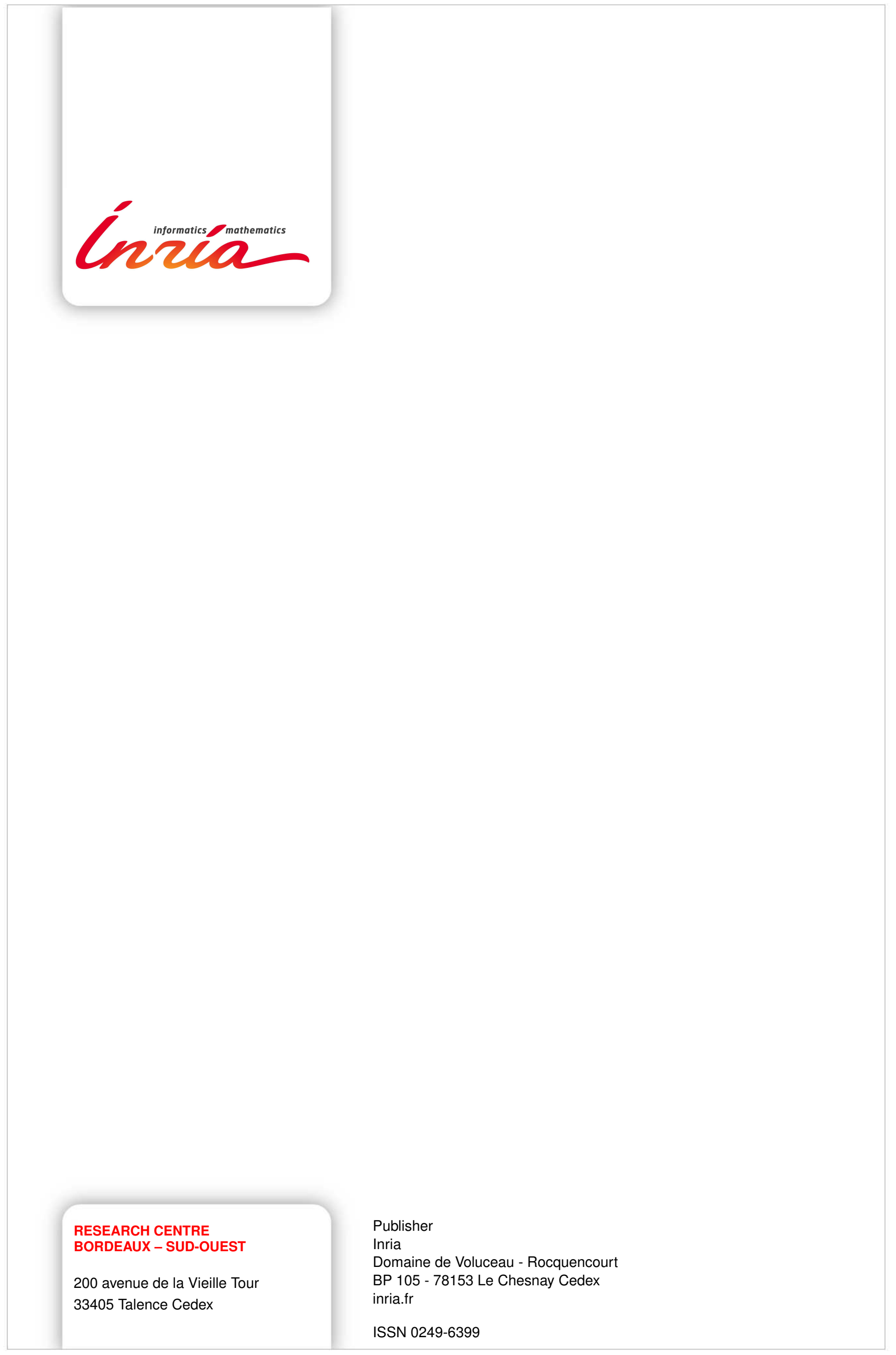

